\title{
PREDiction of AERIAL SpRAY RELEASE FROM UAVS
}

\author{
M. E. Teske, D. A. Wachspress, H. W. Thistle
}

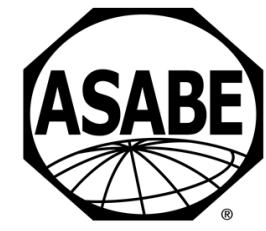

\begin{abstract}
This article summarizes the ability of CHARM+AGDISP to predict the drift and deposition of sprays released from rotary wing unmanned aerial vehicles (UAVs). This predictive capability results from merging algorithms for spray transport, as found in AGDISP (AGricultural DISPersal), with CHARM (Comprehensive Hierarchical Aeromechanics Rotorcraft Model). The resulting software tracks the release of spray droplets from nozzles on the UAV to deposition on the ground. To date, both AGDISP and CHARM, a code that provides a complete representation of the time-varying, unsteady flow field surrounding a helicopter during transient maneuvering flight near the ground, have been extensively validated. The CHARM+AGDISP software is applied to two UAVs to explore the flow field regimes that present challenges for effective UAV operations. The simulations undertaken indicate flight conditions that yield acceptable deposition levels and minimize drift; inversely, conditions are also identified that result in off-target drift that may be problematic.
\end{abstract}

Keywords. Aerial application, AGDISP, CHARM, Helicopter modeling, Unmanned aerial vehicle (UAV).

$\mathrm{T}$ The last five years have seen a dramatic increase in the use of UAVs, either as replacements for larger piloted aircraft or as more applicable, cost-effective options in critical spraying scenarios (e.g., for high efficiency and effective spraying of "spots," small fields, hilly terrain, or high-value crops). While UAVs were initially used for surveillance (Watts et al., 2012; Pajares, 2015; Muchiri and Kimathi, 2016), their evolving use today as spraying platforms (Huang et al., 2009, 2015; Giles et al., 2016) raises questions about their impact and adequacy with regard to deposition and drift of released pesticide products (Faiçal et al., 2014; Xue et al., 2014, 2016; Qin et al., 2016; Tang et al., 2017; Wang et al., 2017). Maximizing in-swath deposition levels while limiting off-target drift by UAVs, which are more maneuverable than full-sized aircraft and can safely fly at much lower altitudes, will require rules with regard to swath offset and limiting meteorological conditions, much like fullsized aircraft, including real-time adjustment of the flight path and on/off options for individual spray nozzles (as currently available on ground sprayers). The question is whether computational tools are available to make real-time predictions of

\footnotetext{
cc) $\$$ The authors have paid for open access for this article. This (c) BY NG ND work is licensed under a Creative Commons AttributionNonCommercial-NoDerivatives 4.0 International License https://creativecommons.org/licenses/by-nc-nd/4.0/

Submitted for review in November 2017 as manuscript number MS 12701; approved for publication by the Machinery Systems Community of ASABE in April 2018.

Mention of company or trade names is for description only and does not imply endorsement by the USDA. The USDA is an equal opportunity provider and employer.

The authors are Milton E. Teske, Senior Associate, and Daniel A. Wachspress, Senior Associate, Continuum Dynamics, Inc., Ewing, New Jersey; Harold W. Thistle, Program Manager, USDA Forest Service, Morgantown, West Virginia. Corresponding author: Milton E. Teske, Continuum Dynamics, Inc., 34 Lexington Ave., Ewing, NJ 08616; phone: 609-538-0444; milt@continuum-dynamics.com.
}

spray deposition in these situations. To this end, the USDA Forest Service and its state and federal cooperators, including the National Aeronautics and Space Administration (NASA) and several branches of the U.S. Department of Defense (DOD), have investigated the application of two well-established numerical tools for the modeling of UAV spraying and its impact on deposition and drift. The combination of these two models, i.e., a proven helicopter flow field model (CHARM) with a proven spray deposition model (AGDISP), is the subject of this article.

\section{APPROACH}

Two models are considered here. Both codes were developed with ongoing support from the USDA Forest Service (AGDISP), NASA, and DOD (CHARM).

\section{AGDISP}

The origin of AGDISP (AGricultural DISPersal) can be traced to 1979 when NASA supported the initial development of a Lagrangian droplet trajectory model to simulate the aerial release of pesticides. This model was based on previous experience in understanding the physics of vortex wakes behind aircraft. A simple vortex wake model, patterned after the approach suggested by Reed (1953), and the subsequent development of a closure technique to recover the effects of atmospheric turbulence on the variance of released spray material about its mean trajectory (von Kármán and Howarth, 1938; Houbolt et al., 1964), led to the first version of AGDISP (Bilanin et al., 1989). Further implementation, funded by the USDA Forest Service (Teske et al., 2003, 2011) led to its current version, AGDISP 8.29. This version of AGDISP includes the Tier I curves from AgDRIFT (Bird et al., 2002; Teske et al., 2002), a sister model to AGDISP. Validation of the model to approximately $800 \mathrm{~m}$ downwind (Teske and Thistle, 2003) opened the door for improved solution handoff to mesoscale 
atmospheric transport models (Allwine et al., 2002; Teske, 2016).

AGDISP includes a simplified helicopter wake model that transitions from downwash under a single set of rotor blades to fully rolled-up tip vortices. This model partitions vehicle weight between hover downwash and rotor tip vortices as a function of time. The hover downwash model is taken from actuator disk theory for a propeller (Bramwell, 1976) and may be written as:

$$
w_{d}=\frac{1}{R} \sqrt{\frac{F W}{2 \pi \rho_{a}}}
$$

where $w_{d}$ is the downwash velocity $\left(\mathrm{m} \mathrm{s}^{-1}\right)$ at the rotor plane, $R$ is the rotor radius $(\mathrm{m}), W$ is the average spraying weight $(\mathrm{N}), \rho_{a}$ is the density of air $\left(\mathrm{g} \mathrm{cm}^{-3}\right)$, and $F$ is a modeling parameter (dimensionless). The strength of the vortex pair may be found from:

$$
\Gamma=\frac{2(1-F) W}{\pi \rho_{a} R U}
$$

where $\Gamma$ is the vortex strength $\left(\mathrm{m}^{2} \mathrm{~s}^{-1}\right)$ and $U$ is the forward speed $\left(\mathrm{m} \mathrm{s}^{-1}\right)$. In the AGDISP model, $F=\exp (-X / R)$, which is found by matching the behavior of this simple model with detailed helicopter model predictions (Wachspress et al., 2003a) as a function of the axial distance $X(\mathrm{~m})$. At the beginning of the calculation $X=0, F=1$, and the weight of the helicopter provides downwash through the rotor blades. As the calculation proceeds, $X>0, F \rightarrow 0$, and the weight transitions to provide vortex motion identical to that of a fixedwing aircraft. Because of the exponential decay, the transition between downwash and vortex motion occurs within two rotor diameters behind the helicopter.

Unfortunately, the AGDISP helicopter model (described by eqs. 1 and 2) is restrictive in two ways: (1) it can only be applied to aircraft with a single main rotor, and (2) the aircraft flying height and speed must be sufficiently high that the downwash model rolls up into a pair of vortices before ground impact by the rotor downwash. These restrictions prevent the existing helicopter model from simulating the behavior of UAV wakes because UAVs often have multiple rotors and fly much closer to the ground and at much lower speeds than helicopters. One way to predict UAV wakes, as described in this article, is to resolve the multiple rotor wakes with a more accurate model, such as CHARM.

\section{CHARM}

CHARM is a self-contained rotor/wake/body computational analysis that models rotorcraft blade aerodynamics and dynamics in hover and forward flight. The formulation and validation of its core rotorcraft analyses are documented by Quackenbush et al. (1990, 1991, 1994, 1996). CHARM delivers highly accurate solutions over a broad range of applications and is designed with a hierarchical structure to enable a single code to be applicable to a full range of modeling tasks, from the prediction of extremely high-resolution airloads to flight dynamics applications, including real-time rotor/wake/body solutions suitable for engineering flight simulation and handling qualities analyses. The major features of the CHARM code are the following:

- The full-span, freely distorting, constant vorticity contour (CVC) wake model and analytical tip vortex roll-up model combine to form a highly sophisticated wake model. Details of the rotor wake are determined from first principles, bypassing the need to provide empirical constants in assigning tip vortex characteristics.

- Fast vortex and fast panel analyses incorporated into the model allow for extremely high-resolution modeling of flow field characteristics and wake/surface interactions in minimal computational time.

- A hierarchy of solution methods allows a tradeoff between resolution and increased computational speed. In this way, a single, consistent modeling architecture can be applied to a wide range of applications.

- The multiple rotor and lifting panel capabilities of the model combine to allow a complete inviscid analysis of complex modern rotorcraft and UAVs, including blade, wake, fuselage, and wing.

- Evolution of the aerodynamics and blade dynamics solutions simultaneously in time can solve for non-periodic solutions, capturing rotor responses to time-varying control inputs.

A complete description of the CHARM code is provided by Quackenbush et al. (1999). The combination of CHARM+AGDISP will enable rapid deposition predictions that render the combined codes suitable for control of drift during aerial application.

A typical wake prediction is shown in figure 1. The trailed vorticity from the tips of the rotor blades is traced by red curves, transitioning from the tips to light blue for inboard vorticity and to dark blue curves for the rotor hub. The same color pattern applies to the tail rotor.

Several features of this model are particularly advantageous for real-time prediction of the aerial application of pesticides by UAVs. First, the flow field solution has been extensively validated for accurate modeling of complete rotorcraft aerodynamics and dynamics in steady and maneuvering flight (Wachspress et al., 2003a, 2008). CHARM can optionally include multiple rotors and the vehicle fuselage, replicating the many UAV configurations seen in the literature. Extensive work has also been performed to accelerate the wake solution for real-time applications. The CHARM wake model originated the use of hierarchical fast vortex (HFV) methods in rotorcraft applications (Wachspress et al., 2003b). HFV methods automatically adapt to any transient maneuver, including an aircraft flying into its "old" wake (as occurs in vertical descent), vortex ring state, and ground vortex flows (which impact UAV operations). Prior research and development saw further acceleration of HFV methods and other areas of the wake solution to support real-time operation while maintaining maximum possible fidelity (Quackenbush et al., 2001). This prior work includes extensive validation of the real-time solution accuracy (Wachspress et al., 2008).

CHARM couples a vortex lattice blade model, a free-vortex wake model, and a fast panel fuselage model to model the full aircraft and its wake effects from first principles, in- 


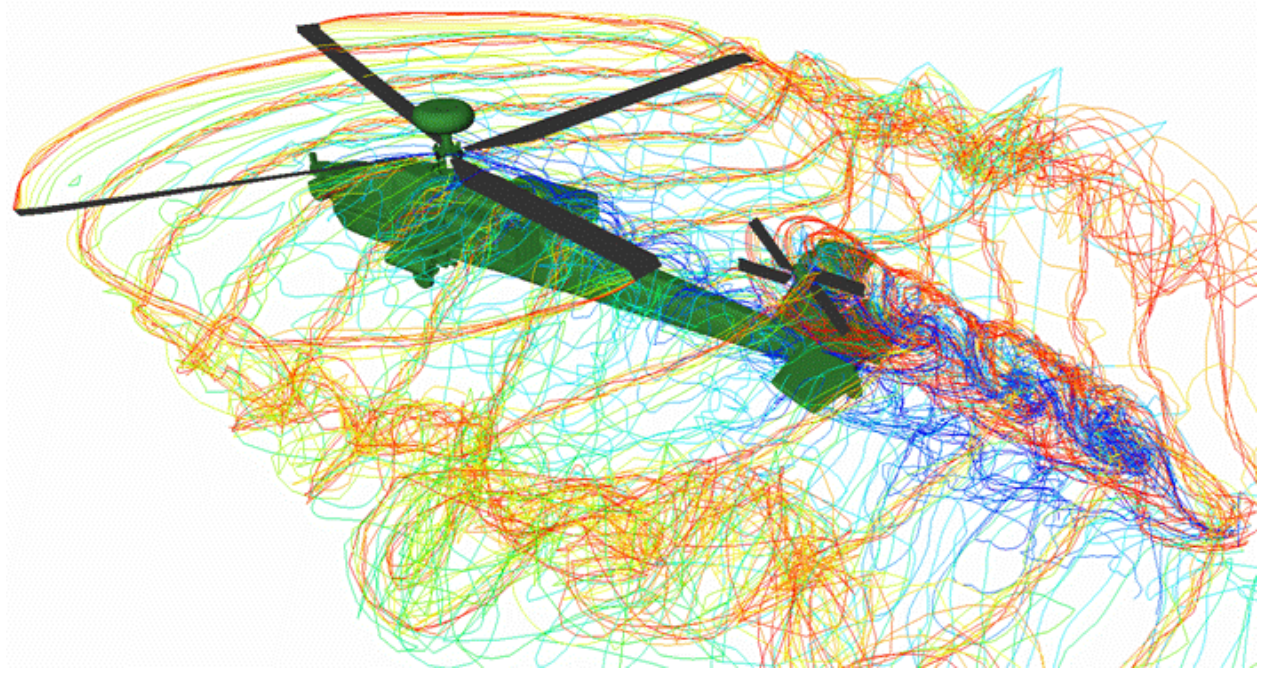

Figure 1. CHARM full-span, free-wake model of the AH-64D Apache helicopter. Tip vorticity (in red) forms a circular wake that interacts with the inboard vorticity (in light blue) to create a complex wake structure that subsequently interacts with the wake from the tail rotor (from Wachspress et al., 2008).

cluding multiple rotors (Wachspress et al., 2012a). The ability of the model to predict rotor outwash in flight near the ground has been extensively validated through correlation with test data, including data generated by tilt rotors (Keller and Wachspress, 2012). CHARM has been used by the U.S. Navy to model the outwash impact of military helicopter wakes on ground personnel, and it has been used by the U.S. Army to model the impact of rotor wakes on ordnance trajectories and brownout (Wachspress et al., 2009). The current model has also been used for real-time simulations (Wachspress et al., 2008, 2012b), but the inclusion of the aerial release of pesticides and the real-time prediction of the subsequent motion of the released spray material have not been discussed until now.

\section{METHOD}

This article details the release of spray material from UAVs, including the effects of spray deposition by AGDISP. CHARM develops the 3D velocity flow field, and AGDISP tracks the release of droplets through this field. The flow field is accurate because CHARM models the physics of the major wake interactional aerodynamics from multiple rotors and has been verified; the deposition and drift are accurate because of the use of the verified Lagrangian droplet model in AGDISP. The two codes are coupled together by the replacement of a single subroutine in AGDISP (which computes the velocity flow field) with the detailed calculations in CHARM.

The problem presented here will discuss two test platforms (fig. 2), the Dragonfly DP-12 Rhino tandem rotor UAV and the Aeronavics ICON octocopter, for one flight condition each. It is not within the scope of this article to describe the impact of either UAV wake on the deposition of released spray material under all possible flight conditions. Instead, it is the intent of this article to illustrate the potential impact of each UAV wake on aerial application under one set of modeling conditions.

An overview comparison between vehicles is shown in table 1, which includes corresponding values for the Bell 206B JetRanger II, a popular spray helicopter. More specific details on both UAVs are provided by Wachspress (2015) and Teske and Wachspress (2017).

The DP-12 calculations placed the spray boom $1 \mathrm{~m}$ below the main (front) rotor hub (fig. 2a), extending the boom out to roughly $1 \mathrm{~m}$ on either side of the UAV, with nine evenly spaced nozzles positioned on the boom. The spray release height was set to $1.5 \mathrm{~m}$ above the ground; a swath width of $3 \mathrm{~m}$ was assumed while tracking the released spray material from the nozzles. The ICON calculations were made to demonstrate spraying strategies for an aircraft that is much lighter than the DP-12. In these calculations, the spray boom was placed $0.5 \mathrm{~m}$ below the lower blades (in the middle between the blades for balance, fig. 2b), extending the boom out to roughly $0.75 \mathrm{~m}$ on either side of the UAV, with eleven evenly spaced nozzles positioned on the boom. The spray release height was set to $1.5 \mathrm{~m}$ above the ground; a swath width of $3 \mathrm{~m}$ was assumed while tracking the released spray material from the nozzles.

For the spray release height assumed here, each AGDISP calculation on a personal computer took 1 to $5 \mathrm{~s}$, while the same calculation with CHARM+AGDISP took 1 to $2 \mathrm{~min}$.

Plotted results include four application parameters of interest: (1) coefficient of variation, (2) application efficiency, (3) downwind deposition, and (4) airborne drift. The coefficient of variation (COV) is a measure of how uniformly spray material deposits across the swath; smaller COV values suggest a more even spray deposition pattern on the ground. Typically, a COV of 0.3 or less is considered desirable (Parkin and Wyatt, 1982). Application efficiency is a measure of how much spray material lands within the swath. If the application efficiency is 1.0, all active ingredients in the spray have deposited within the swath; contrarily, if the application efficiency is 0.0 , none of the active ingredients landed there. Downwind deposition is the fraction of active ingredients deposited downwind of the swath, while airborne drift is the fraction of active ingredients still airborne at a specified downwind distance. For these calculations, the 


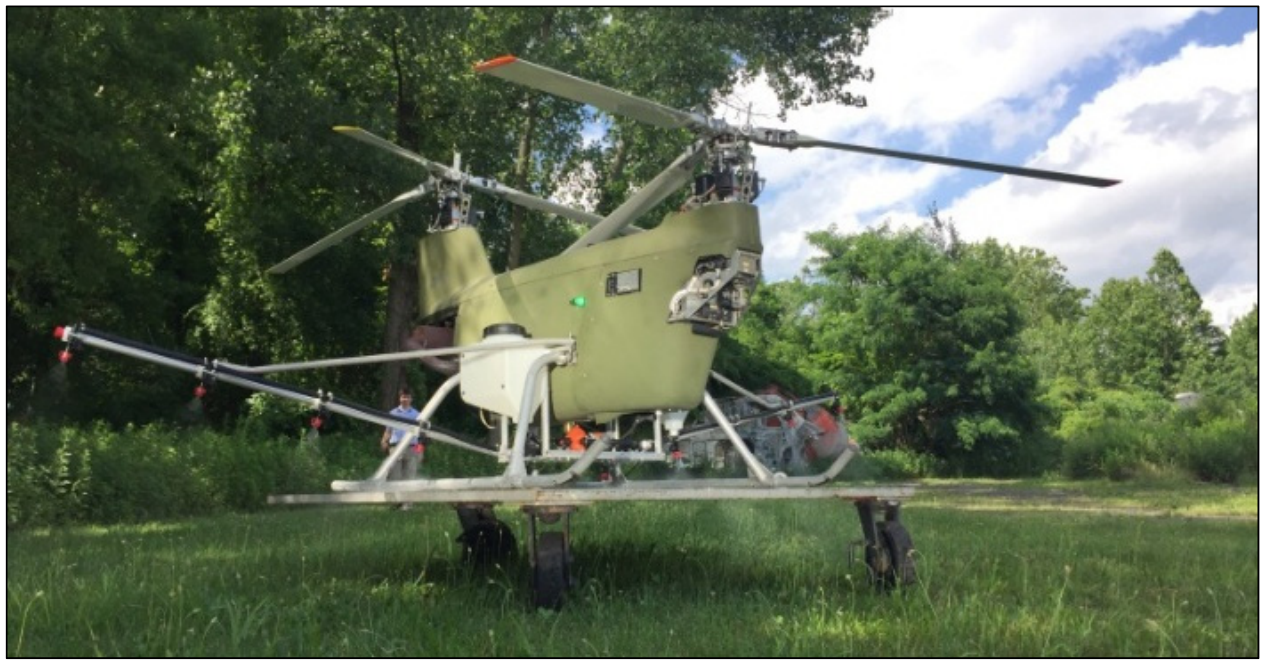

(a)

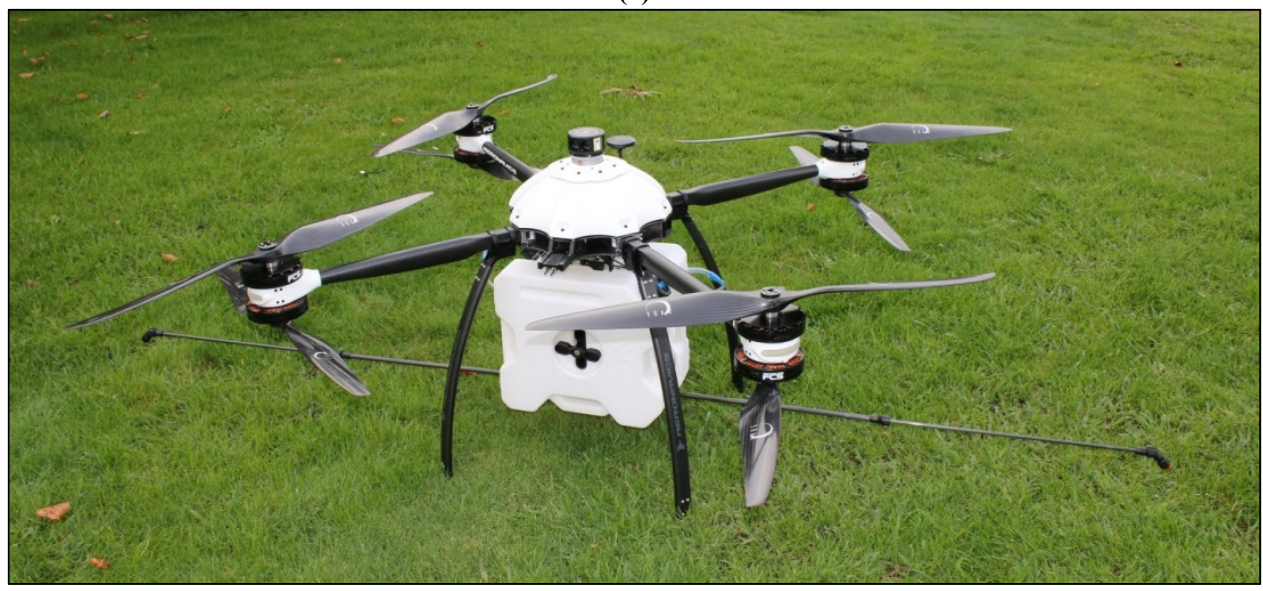

(b)

Figure 2. (a) Dragonfly DP-12 Rhino tandem rotor and (b) Aeronavics ICON octocopter.

downwind distance was set to $6.0 \mathrm{~m}$ downwind of the downwind edge of the swath.

Table 1. Comparison of aircraft and UAV characteristics.

\begin{tabular}{cccc}
\hline & Bell 206B & Dragonfly & Aeronavics \\
JetRanger II & DP-12 Rhino & ICON \\
\hline Rotor radius $(\mathrm{m})$ & 5.08 & 1.37 & 0.39 (avg.) \\
Number of rotors & 1 & 2 & 8 \\
Spraying weight $(\mathrm{kg})$ & 1000.0 & 220.0 & 30.0 (avg.) \\
Spraying speed $\left(\mathrm{m} \mathrm{s}^{-1}\right)$ & 30.8 & 5.0 to 15.0 & 10.0 to 20.0 \\
Rotation rate $\left(\mathrm{rpm}^{-}\right)$ & 384 & 1050 & 2800 (avg.) \\
Downwash $\left(\mathrm{m} \mathrm{s}^{-1}\right)$ & 7.0 & 8.6 & 5.6 \\
\hline
\end{tabular}

\section{RESULTS}

The intent of this article is to demonstrate spraying strategies for the two UAVs based on calculations made with the CHARM+AGDISP code. Consequently, both UAVs were simulated across a wide range of forward speeds, operating under nominal flight conditions, including meteorological

Table 2. UAV application parameters investigated.

\begin{tabular}{ccc}
\hline & Dragonfly & Aeronavics \\
Parameter & DP-12 Rhino & ICON \\
\hline Release height $(\mathrm{m})$ & 1.5 & 1.5 \\
Swath width $(\mathrm{m})$ & 3.0 & 3.0 \\
Number of nozzles & 9 & 11 \\
Droplet sizes $(\mu \mathrm{m})$ & 100.0 & $100.0,300.0,500.0$ \\
Spraying speeds $\left(\mathrm{m} \mathrm{s}^{-1}\right)$ & 4.0 to 14.0 & 2.0 to 12.0 \\
Crosswind speeds $\left(\mathrm{m} \mathrm{s}^{-1}\right)$ & 0.22 and 4.47 & 0.22 and 2.24 \\
\hline
\end{tabular}

and payload levels. Only monosized droplets were tracked by the codes. Table 2 summarizes the application parameters for both UAVs. The constant meteorological conditions assumed reflect the default input conditions in AGDISP. Vortical flow fields generated by the UAVs were initiated by counter-rotating rotors. Looking down at the DP-12, the front rotor rotates counterclockwise, and the rear rotor rotates clockwise. Looking down at the ICON, the front left rotor rotates clockwise, the front right rotor rotates counterclockwise, the rear left rotor rotates counterclockwise, and the rear right rotor rotates clockwise. The four rotors below the top set of rotors rotate opposite to the rotors above them. It may be seen from figure 3 that the tandem wakes of the DP-12 and the multiple wakes of the ICON generate complex wake interactions.

\section{DP-12 DEMONSTRATION SPRAYING STRATEGIES}

Results for the DP-12 are shown in figure 4 for two crosswinds: 0.22 and $4.47 \mathrm{~m} \mathrm{~s}^{-1}$. Figure $4 \mathrm{a}$ shows the application properties for the low crosswind $\left(0.22 \mathrm{~m} \mathrm{~s}^{-1}\right)$. It is seen that there is no airborne drift, and the application efficiency is essentially 1.0. Thus, under very low crosswind conditions, the spray remains within the swath (higher release heights tend to allow spray droplets to move downwind, as presented 


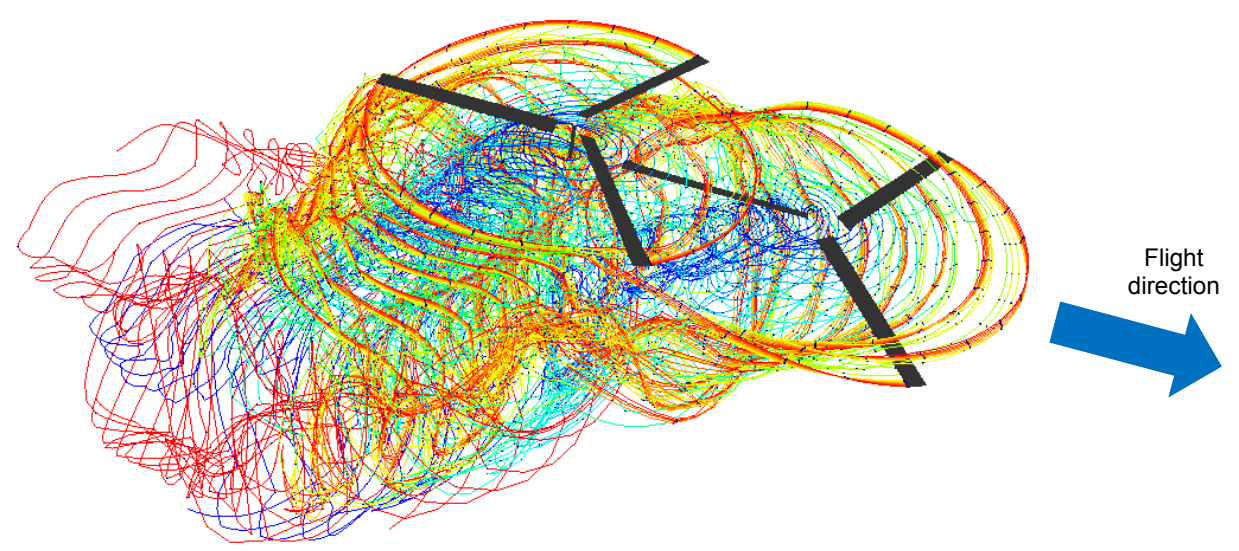

(a)

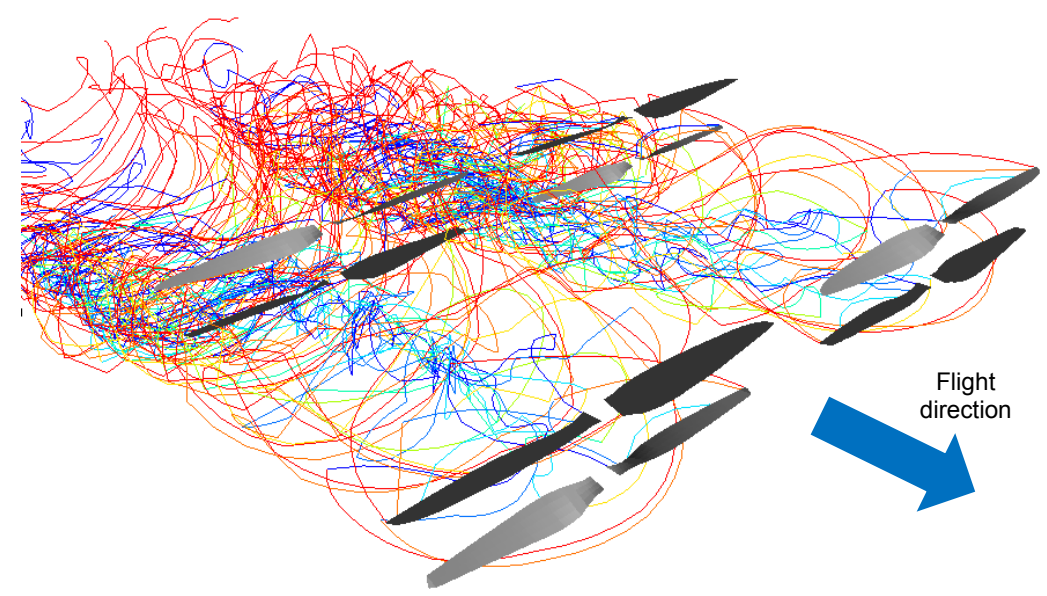

(b)

Figure 3. Wake behavior for the (a) Dragonfly DP-12 Rhino tandem rotor and (b) Aeronavics ICON octocopter. The colors of the trailed vorticity are identical to that shown in figure 1 for the AH-64D Apache helicopter. The blue arrows are the UAV flight directions. These drawings are not to the same scale, as the rotor blades on the DP-12 are 3.5 times larger than the rotor blades on the ICON (see table 1 for size comparison). The stacked rotor blades on the ICON rotate in opposite directions.

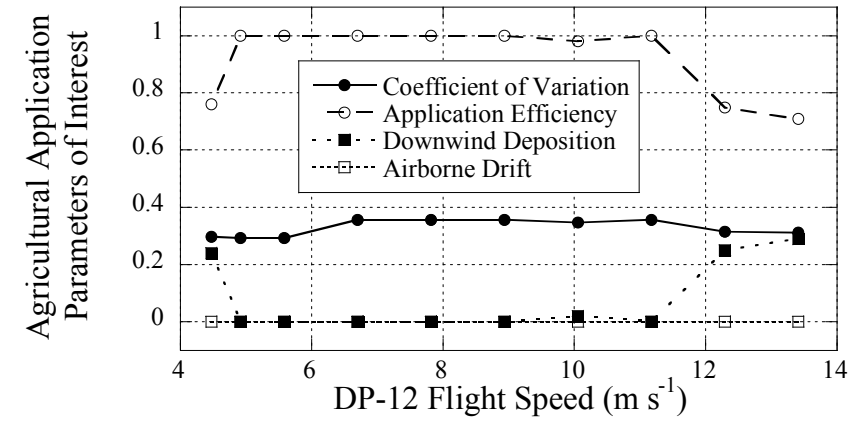

(a)

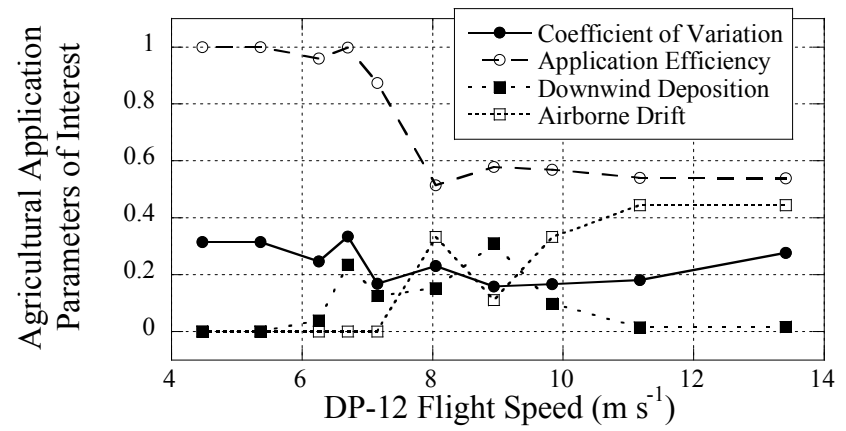

(b)

Figure 4. CHARM+AGDISP predictions of agricultural application parameters of interest for the DP-12 for two ambient crosswinds: (a) $0.22 \mathrm{~m}$ $\mathrm{s}^{-1}$ and (b) $4.47 \mathrm{~m} \mathrm{~s}^{-1}$.

in the Discussion section). Figure $4 \mathrm{~b}$ (with the high crosswind of $4.47 \mathrm{~m} \mathrm{~s}^{-1}$ ) shows that the application properties are similar to figure 4a until the flight speed reaches $6.5 \mathrm{~m} \mathrm{~s}^{-1}$. Higher flight speeds increase airborne drift by capturing spray droplets and keeping them aloft.

The decrease in application efficiency between 6.5 and $8 \mathrm{~m} \mathrm{~s}^{-1}$ (fig. 4b) occurs as the flight speed nears the critical speed $\left(V_{\text {crit }}, \mathrm{m} \mathrm{s}^{-1}\right)$, which is approximately equal to the hover downwash speed $\left(w_{d}\right)$ obtained from equation 1 with $F=1$.
Values for the Bell 206B, DP-12 and ICON are given in table 1, where it may be seen that the critical speed for the DP-12 is approximately $8.6 \mathrm{~m} \mathrm{~s}^{-1}$.

A plan view of the DP-12 wake is shown in figure 5, illustrating the wide spread of the vortical wake and the deposition position of the released droplets. Note that the deposition is not centered directly behind the aircraft, even though the spray droplets were released symmetrically from the boom, because of the effect of the crosswind. 


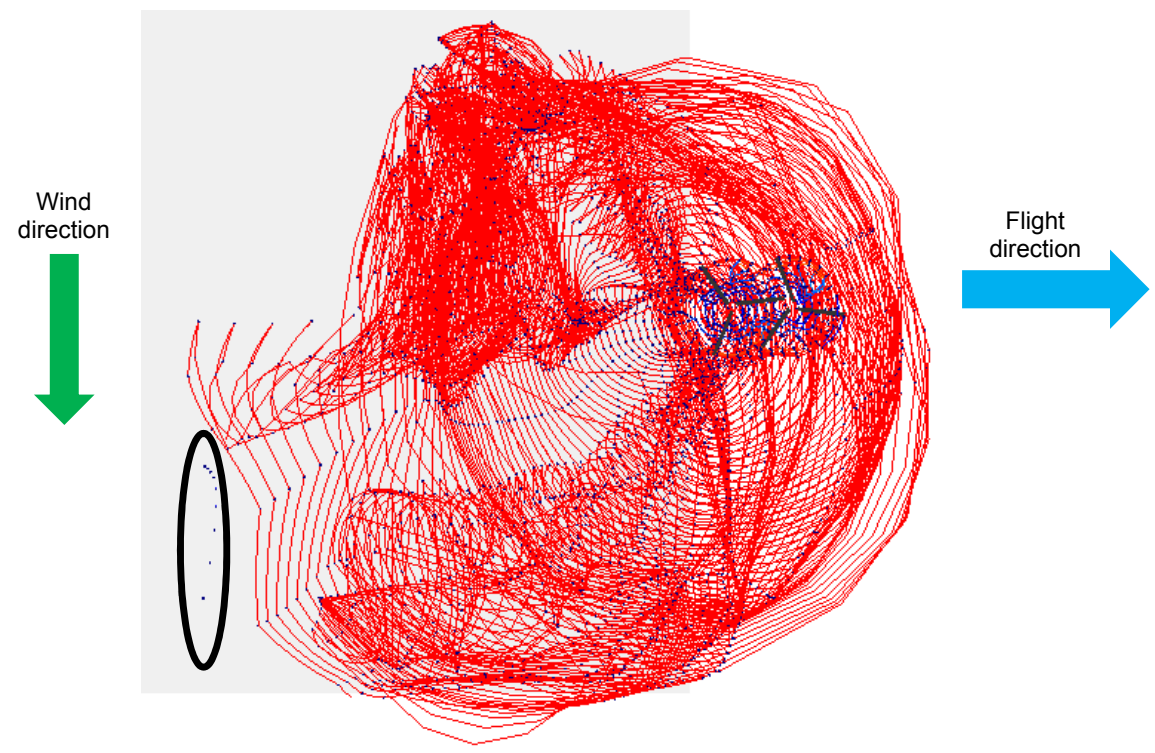

Figure 5. CHARM+AGDISP predictions of the DP-12 wake for a flight speed of $8.94 \mathrm{~m} \mathrm{~s}^{-1}$ in a crosswind of $4.47 \mathrm{~m} \mathrm{~s}^{-1}$. The ellipse in black (lower left behind the UAV) identifies the ground locations of the spray material released from each of the nine nozzles. The location of the UAV is identified by the blue rotor blades near the front of the vortical wake (somewhat obscured by the wake). The spray boom is positioned under the forward rotor blades. The flight direction of the DP-12 is shown by the blue arrow, and the wind direction is shown by the green arrow. Only the blade tip vortices are shown (in red) for clarity.

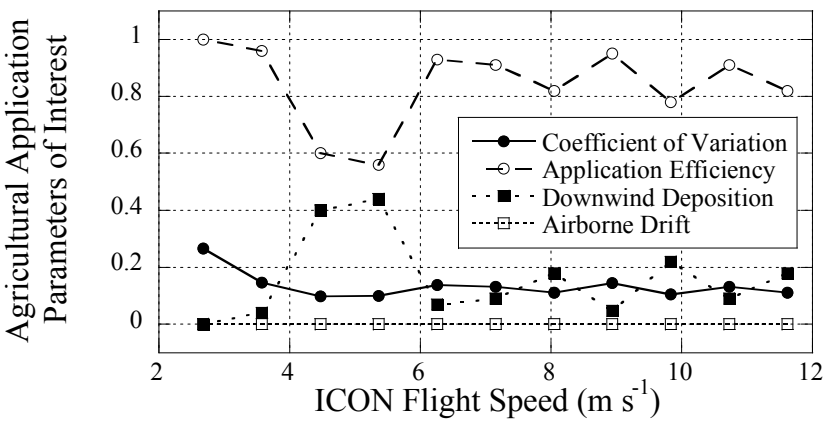

(a)

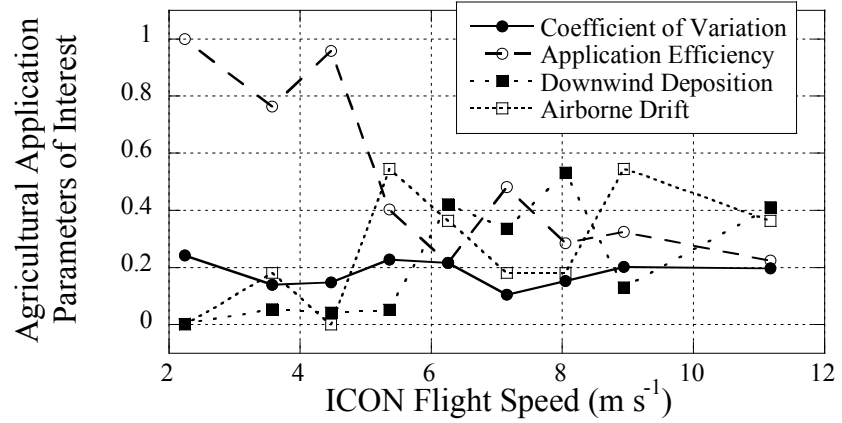

(b)

Figure 6. CHARM+AGDISP predictions of agricultural application parameters of interest for the ICON for two ambient crosswinds: (a) $0.22 \mathrm{~m} \mathrm{~s}^{-1}$ and (b) $2.24 \mathrm{~m} \mathrm{~s}^{-1}$.

\section{ICON DEMONSTRATION SPRAYING STRATEGIES}

Results for the ICON are shown in figure 6 for two crosswinds: 0.22 and $2.24 \mathrm{~m} \mathrm{~s}^{-1}$. The plotted results include the same four application parameters of interest: COV, application efficiency, downwind deposition, and airborne drift. It is seen that the lighter ICON octocopter and the much heavier DP-12 give somewhat similar results, especially at the higher crosswind speeds. Downwash from the ICON is not as strong, and therefore the critical speed below which the ICON should operate is lower than for the DP-12, approximately $5.6 \mathrm{~m} \mathrm{~s}^{-1}$ (table 1). Application efficiency decreases rapidly between 4.5 and $6 \mathrm{~m} \mathrm{~s}^{-1}$ (fig. $6 \mathrm{~b}$ ). Calculations to date suggest that the ICON would need to operate in minimal crosswind and at very low flight speeds to be an effective spraying UAV.

A plan view of the ICON wake is shown in figure 7, indicating the narrow distribution of the wake and the deposition position of the droplets released from the UAV. Again, this deposition is displaced because of the crosswind.

\section{DiscUSSION}

The two examples shown here demonstrate the computational ability of CHARM+AGDISP for the two UAVs examined, especially with regard to the levels of drift predicted. As with full-sized fixed-wing aircraft and helicopters, the pilot operating the UAVs would be expected to understand the effects of crosswind and adjust the UAV flight path. This corrective action would tend to improve the application efficiency and reduce the downwind deposition (because of the repositioning of the UAV flight path in response to the crosswind) but may not necessarily improve the COV or reduce airborne drift.

Figure $4 \mathrm{~b}$ shows the DP-12 results for a high crosswind of $4.47 \mathrm{~m} \mathrm{~s}^{-1}$. In this case, the application efficiency is poor at flight speeds higher than $6.5 \mathrm{~m} \mathrm{~s}^{-1}$, which is lower than the DP-12's critical speed. Figure $6 \mathrm{~b}$ shows a loss of drift control with the ICON at flight speeds higher than $4.5 \mathrm{~m} \mathrm{~s}^{-1}$ (lower than its critical speed) for a crosswind of $2.24 \mathrm{~m} \mathrm{~s}^{-1}$. In both cases, the deterioration of the application efficiency is quite pronounced, emphasizing the importance of always operating below the critical speed in order to use the rotor downwash to assist in pushing spray material toward the 


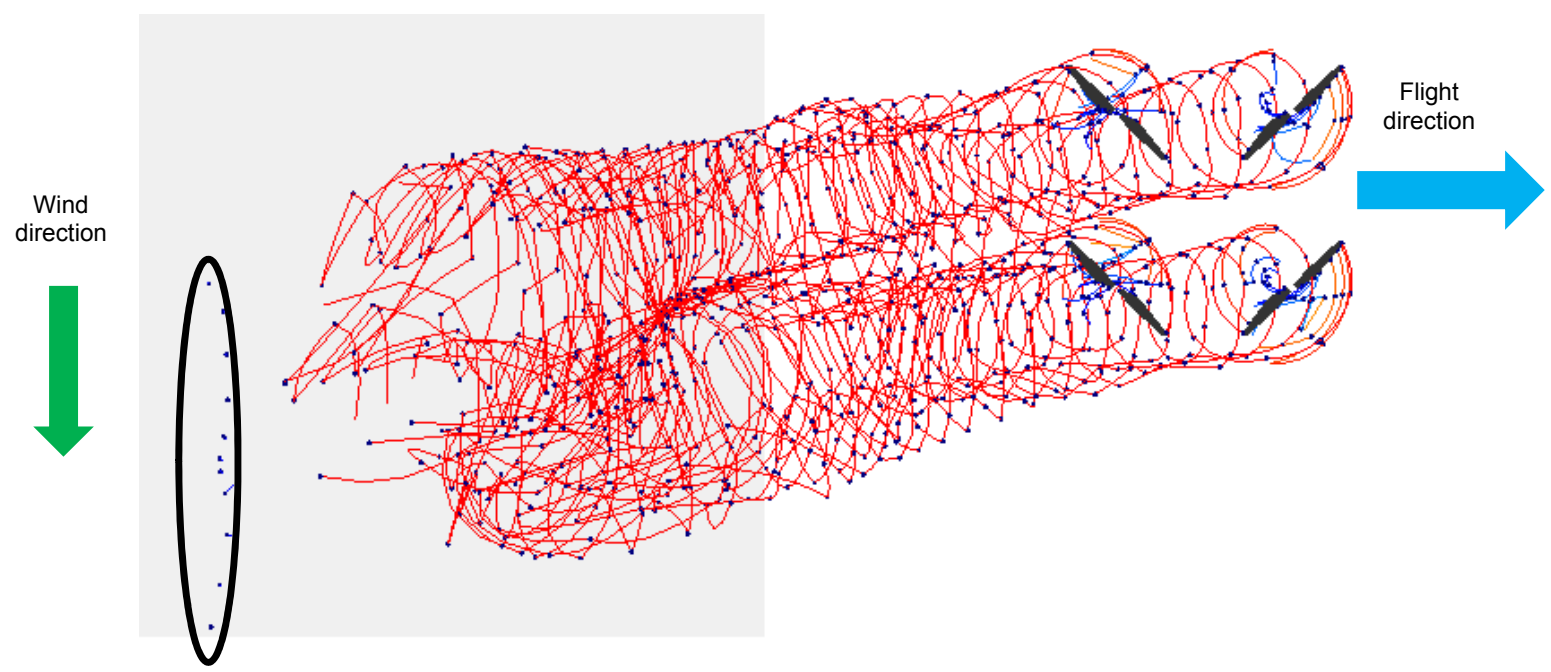

Figure 7. CHARM+AGDISP predictions of the ICON wake for a flight speed of $8.94 \mathrm{~m} \mathrm{~s}^{-1}$ in a crosswind of $2.24 \mathrm{~m} \mathrm{~s}^{-1}$. The ellipse in black (lower left behind the UAV) denotes the locations of the spray material released from each of the eleven nozzles. The location of the UAV is identified by the black rotor blades near the front of the vortical wake. The spray boom is positioned between the rotor blade pairs. The flight direction of the ICON is shown by the blue arrow, and the wind direction is shown by the green arrow. Only the blade tip vortices from the top four blades on the ICON are shown (in red) for clarity.

ground. Otherwise, rotor outwash may entrain the spray upward, resulting in excessive airborne drift.

It must be emphasized that the predictions shown here are for two specific crosswinds $\left(4.47 \mathrm{~m} \mathrm{~s}^{-1}\right.$ for the DP-12 and $2.24 \mathrm{~m} \mathrm{~s}^{-1}$ for the ICON), one release height, one UAV weight for each aircraft analyzed, and one droplet size. To complete the calculations, a variation in release height is
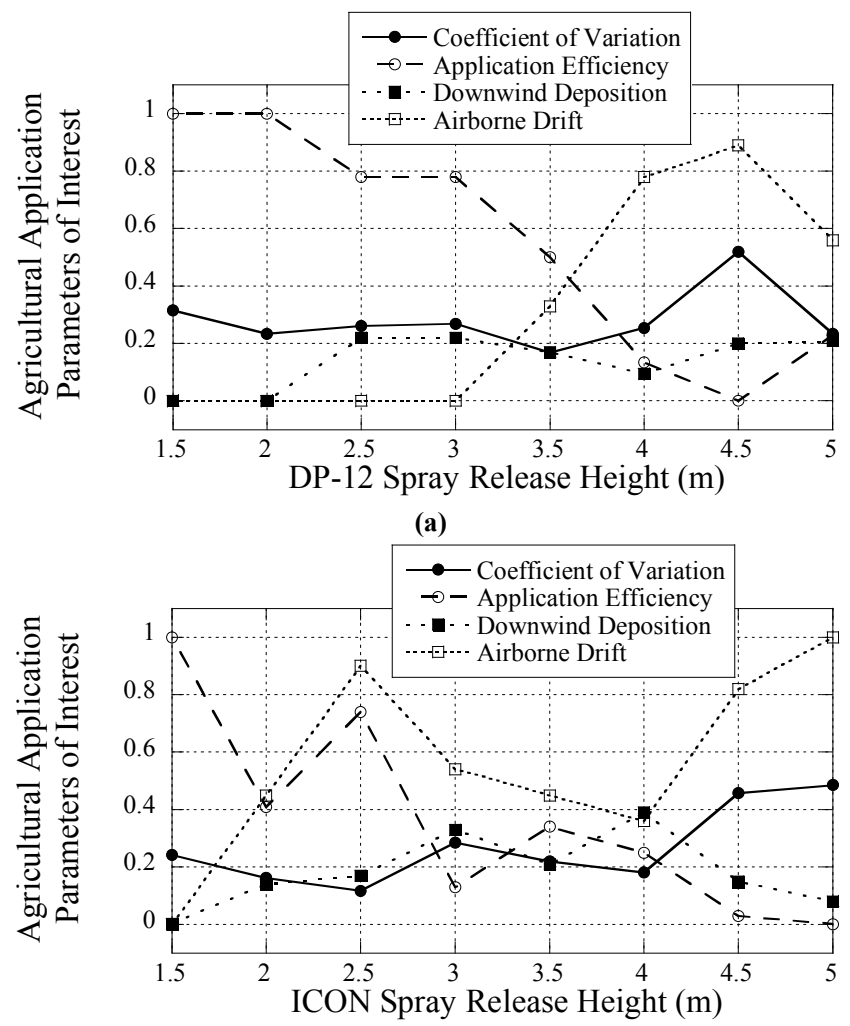

(b)

Figure 8. CHARM+AGDISP predictions of agricultural application parameters of interest for a change in release height with (a) the DP-12 at a flight speed and crosswind of $4.47 \mathrm{~m} \mathrm{~s}^{-1}$ and (b) the ICON at a flight speed and crosswind of $2.24 \mathrm{~m} \mathrm{~s}^{-1}$. shown in figure 8 for a flight speed and crosswind of $4.47 \mathrm{~m}$ $\mathrm{s}^{-1}$ for the DP-12 and $2.24 \mathrm{~m} \mathrm{~s}^{-1}$ for the ICON. Here it may be seen that, as the release height increases, the application efficiency decreases and the airborne drift increases. These effects are expected, based upon numerous sensitivity studies that identify release height as the most important spraying parameter (Teske and Barry, 1993).

Generalizations based on these results are not encouraged. The behavior of the UAV wake for other release heights, crosswind speeds, flight speeds, and UAV weights would have to be quantified through the use of a model or actual flight tests, as would the deposition of complete droplet size distributions. Different release heights alter the transition from downwash through the rotor blades of the UAV to outwash away from the UAV when the ground is reached. Different crosswind speeds alter the effect of the outwash, especially if the crosswind is sufficiently strong so as to impede the velocity of the outwash in the direction opposite to the crosswind direction. Increased flight speeds overtake the velocity of the outwash from the UAV, creating additional problems. Finally, UAV weight, especially for the ICON example shown here, varies as the spray mission continues. As the weight of the UAV decreases, the downwash decreases (eq. 1), reducing its ability to convect droplets to the ground and thereby further increasing airborne drift.

For slow speeds directly under the UAV, the ground impact velocity will be the critical speed defined in equation 3 . For droplets depositing away from the UAV, the ground impact velocity will be the square root of the sum of the squares of the wind speed (which is close to zero) and the terminal velocity of the droplet (which depends on droplet size).

The impact of the UAV wake on droplet deposition can be illustrated by comparing droplet trajectories from the ICON, as shown in figure 9. Three droplet sizes are shown here, representing the effect of the wake on spray material released from the UAV. The droplet paths are clearly chaotic, with several unexpected features: (1) most of the droplets released from the nozzles are pushed erratically around their 


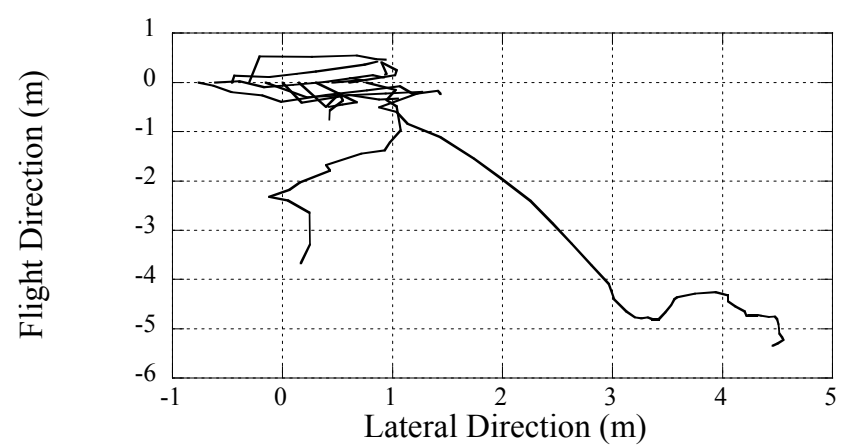

(a)

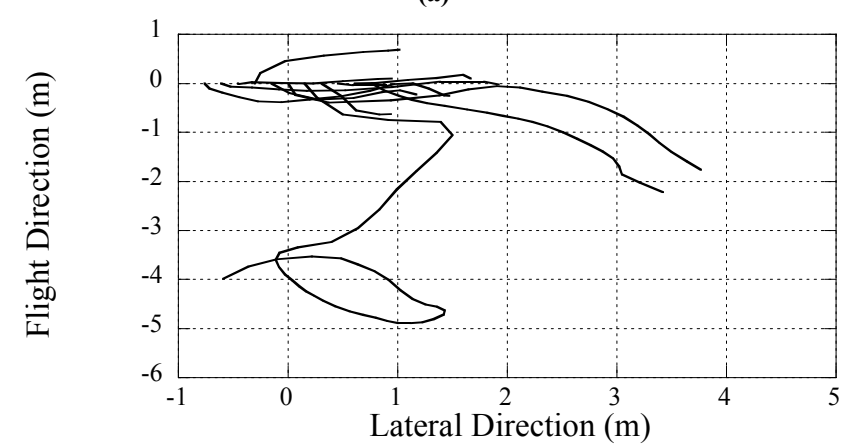

(b)

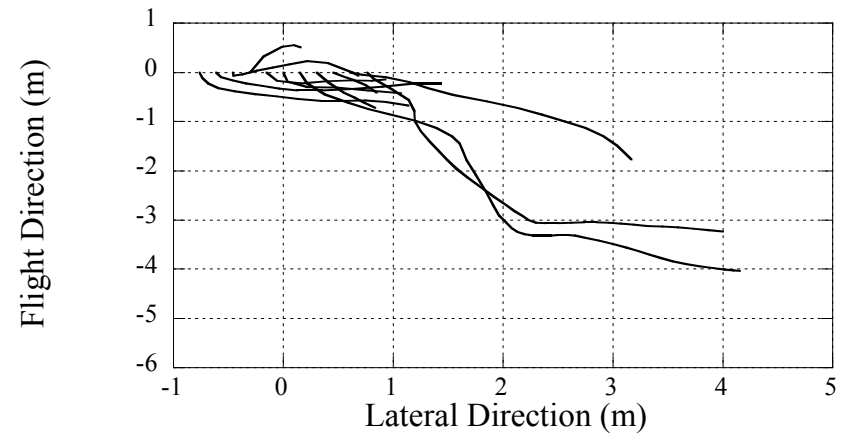

(c)

Figure 9. Trajectories for droplet sizes of (a) $100 \mu \mathrm{m}$, (b) $300 \mu \mathrm{m}$, and (c) $500 \mu \mathrm{m}$ released from the ICON with a flight speed of $2.68 \mathrm{~m} \mathrm{~s}^{-1}$ and a crosswind from left to right of $2.24 \mathrm{~m} \mathrm{~s}^{-1}$. Droplet trajectories end when the droplets reach the ground.

release points before depositing on the ground; (2) the $100 \mu \mathrm{m}$ droplets are not driven aloft by the ICON wake (which is the behavior of this droplet size when released from a full-sized helicopter) but instead deposit within the swath width (-1.5 to $1.5 \mathrm{~m})$, although one droplet does travel downwind; and (3) the 300 and $500 \mu \mathrm{m}$ droplets also deposit within the swath (similar to their behavior when released from a full-sized helicopter), but, interestingly, several of these droplets are driven aloft and transported downwind. It can then be expected that the deposition pattern anticipated with the release of a complete droplet size distribution from the ICON (and possibly from all UAVs) will challenge the paradigm established by full-sized helicopter spraying.

A final comparison is shown in figure 10, plotting the behavior of the COV, application efficiency, downwind deposition, and airborne drift with the single-rotor AGDISP model applied to the DP-12 and the ICON. In this application, the UAV wakes are represented by downwash transitioning to a single pair of oppositely rotating vortices.

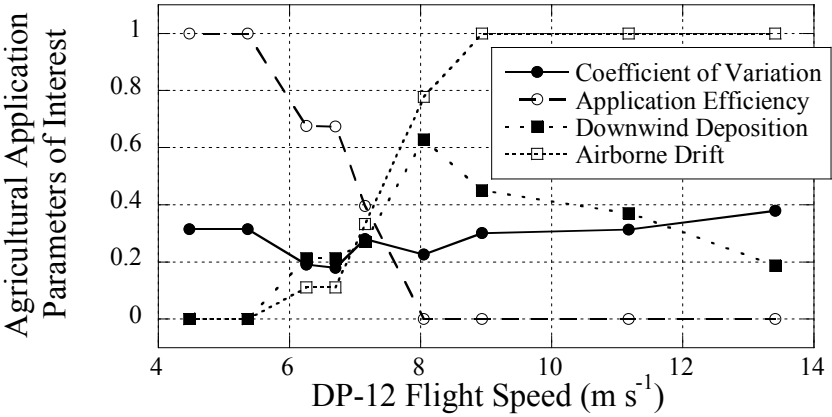

(a)

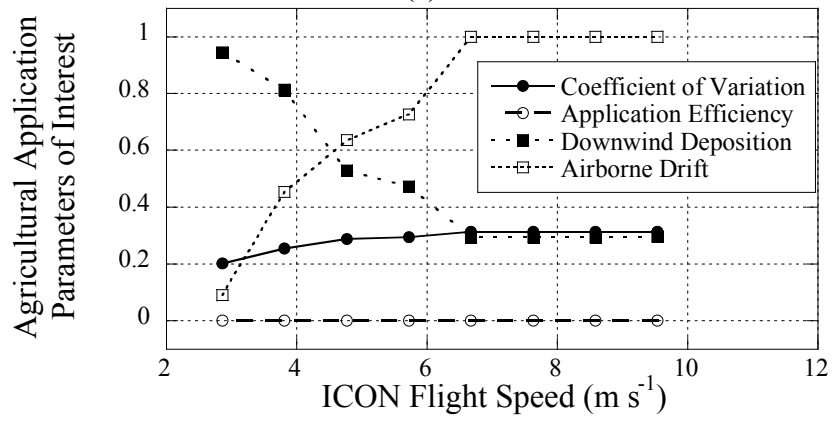

(b)

Figure 10. AGDISP predictions of agricultural application parameters of interest for (a) the DP-12 with an ambient crosswind of $4.47 \mathrm{~m} \mathrm{~s}^{-1}$ and (b) the ICON with an ambient crosswind of $2.24 \mathrm{~m} \mathrm{~s}^{-1}$.

The following observations may be made regarding the predictions shown in figure 10 :

- A comparison of the DP-12 results with CHARM+AGDISP (fig. 4b) and with AGDISP alone (fig. 10a) indicates that the UAV can be flown $25 \%$ faster than predicted by AGDISP alone and still retain high application efficiency.

- A comparison of the ICON results with CHARM+AGDISP (fig. 6b) and with AGDISP alone (fig. 10b) indicates that CHARM+AGDISP predicts acceptable airborne drift and application efficiency at flight speeds up to $80 \%$ of the critical speed, as opposed to the poor results predicted by AGDISP alone.

- It may therefore be seen by comparison of figure 10 with figures $4 \mathrm{~b}$ and $6 \mathrm{~b}$ that use of AGDISP alone will not replicate the spray droplet transport predicted by CHARM+AGDISP for the DP-12 and ICON.

From the results summarized here, a pattern emerges with regard to the effectiveness of spraying with these two UAVs, i.e., the apparent development of a flight envelope that can significantly impact ground deposition levels. It appears that proximity to the ground and/or the flight speed and/or crosswind can cause the released spray material to be lofted above the UAV, producing significant airborne drift. Figures 4 and 6 compare the behavior of the UAVs with regard to deposition effectiveness as a function of flight speed; figure 8 compares the UAVs with regard to the agricultural application parameters of interest as a function of release height. These figures illustrate the operational realities predicted by the CHARM+AGDISP model for the use of UAVs in spraying, i.e., the need for lower boom heights, lower flight speeds, and lower crosswind.

The general trend discovered through the CHARM+AGDISP analysis for UAVs performing spray application is that 
the lower the release height, aircraft speed, and ambient wind, the more uniform, precise, and efficient the spraying process will be. This result is not a surprise, but it can now be quantified. At low flight speeds, the strong downwash beneath the UAV rotors pushes the spray quickly toward the ground and may potentially provide better distribution over individual plants, as opposed to merely coating their upper surfaces. However, as flight speed increases, a critical speed is reached at which the downwash transitions to outwash well before the released droplets reach the ground. As these vortices form, the CHARM+AGDISP solution broadens the vortical field behind the UAV as it expands upward (because the ground prevents expansion downward). If spray material becomes trapped within this developing wake, it could travel to unanticipated distances. At speeds higher than the critical speed, if there is a crosswind, the spray drift may become considerable.

Further, whatever the UAV size, including the "fullsized" aircraft that DOD designates as UAVs (General Atomics' MQ-1 Predators and MQ-9 Reapers, commonly identified as drones), the most important parameter when flying close to the ground is flight speed. If the vehicle is flying slowly enough for ground vortex flow to occur, then greater drift will occur. If the vehicle is flying fast, the wake of the UAV will behave much like that of a full-sized aircraft, negating the usefulness of the UAV for the applications described at the beginning of this article.

Release height therefore plays a significant role in spray deposition. Higher release heights impact the deposition location, in-swath and downwind. The ground impact of released spray as a function of height appears to follow the same patterns for UAVs as for full-sized aircraft. The potential complexity of the UAV wake, i.e., the impact of multiple rotor blades, is the randomizer in this behavior. What is especially critical is to understand the pattern and behavior of the multiple rotor wake and its possible ability to loft released spray droplets, an effect not present with full-sized helicopters because of their higher altitudes, flight speeds, and spray boom positions.

The power of the combined CHARM+AGDISP model is that applicators can now optimize the release height as a function of UAV weight, consider the anticipated wind speed, and then exercise the model to determine the most effective spraying speed (with the lowest flying time), while achieving acceptable deposition levels within the swath and reducing airborne drift. For every UAV rotor blade configuration, there are release height, flight speed, crosswind, and UAV weight limits that the applicator must consider to achieve accurate and successful spraying. Understanding the wake geometry generated by a specific UAV would seem essential to understanding its influence on released spray, considering the response of the complex UAV wake structure to the release height, flight speed, and UAV weight, along with the crosswind imposed. Combining AGDISP with CHARM quantifies, for the first time, the optimum flight speed, release height, and crosswind for any UAV spray application of interest.

\section{CONCLUSIONS}

This article explored the theoretical behavior of two
UAVs (the DP-12 tandem rotor and the ICON octocopter), as representative of UAV size limits, in two spraying scenarios of low and high speed crosswind. The development of the wake motion and deposition potential were predicted by combining the helicopter technology developed in CHARM with the droplet trajectory analysis in AGDISP. The CHARM+AGDISP model demonstrated the importance of a critical flight speed. This speed acts as an upper limit to the forward speed of the UAV irrespective of crosswind or release height (for a given spray boom configuration). Spraying at speeds above the critical speed should be avoided, if possible, as the simulations shown here suggested that spray application effectiveness will be compromised at flight speeds above the critical speed. As a consequence, care should be taken when selecting a UAV for use in a specific spraying scenario. The UAV selection is not as simple as it may appear, nor are the spraying characteristics of a particular UAV obvious. Detailed analyses of the UAV of choice should always be considered.

\section{ACKNOWLEDGEMENTS}

The studies with the CHARM+AGDISP code were supported by NASA at Langley Research Center through the Small Business Innovative Research (SBIR) Program under Contract No. NNX16CL23C, and by the USDA Forest Service, Forest Health Protection MO-FHTET. The authors wish to acknowledge the CORs of the current SBIR effort, Ms. Kelly Hayhurst and Mr. Jeffrey Maddalon, for their assistance and guidance in the performance of this work.

\section{REFERENCES}

Allwine, K. J., Thistle, H. W., Teske, M. E., \& Anhold, J. (2002). The agricultural dispersal-valley drift spray drift modeling system compared with pesticide drift data. Environ. Toxicol. Chem., 21(5), 1085-1090.

https://doi.org/10.1002/etc.5620210526

Bilanin, A. J., Teske, M. E., Barry, J. W., \& Ekblad, R. B. (1989). AGDISP: The aircraft spray dispersion model, code development and experimental validation. Trans. ASAE, 32(1), 327-334. https://doi.org/10.13031/2013.31005

Bird, S. L., Perry, S. G., Ray, S. L., \& Teske, M. E. (2002). Evaluation of the AgDISP aerial spray algorithms in the AgDRIFT model. Environ. Toxicol. Chem., 21(3), 672-681. https://doi.org/10.1002/etc.5620210328

Bramwell, A. R. S. (1976). Helicopter dynamics. Hoboken, NJ: John Wiley and Sons.

Faiçal, B. S., Costa, F. G., Pessin, G., Ueyama, J., Freitas, H., Colombo, A., ... Braun, T. (2014). The use of unmanned aerial vehicles and wireless sensor networks for spraying pesticides. $J$. Syst. Archit., 60(4), 393-404. https://doi.org/10.1016/j.sysarc.2014.01.004

Giles, D. K., Billing, R., \& Singh, W. (2016). Performance results, economic viability, and outlook for remotely piloted aircraft for agricultural spraying. Aspects Appl. Biol., 132, 15-21.

Houbolt, J. C., Steiner, R., \& Pratt, K. G. (1964). Dynamic response of airplanes to atmospheric turbulence including flight data on input and response. NASA TR R-199. Washington, DC: National Aeronautics and Space Administration.

Huang, Y., Hoffman, W. C., Lan, Y., Fritz, B. K., \& Thomson, S. J. (2015). Development of a low-volume sprayer for an unmanned helicopter. J. Agric. Sci., 7(1), 148-153.

Huang, Y., Hoffmann, W. C., Lan, Y., Wu, W., \& Fritz, B. K. (2009). Development of a spray system for an unmanned aerial 
vehicle platform. App. Eng. Agric., 25(6), 803-809. https://doi.org/10.13031/2013.29229

Keller, J. D., \& Wachspress, D. A. (2012). Validation of the CHARM aerodynamic module for engineering and piloted simulation of next-generation tilt rotor aircraft. Report No. 1203. Ewing, NJ: Continuum Dynamics, Inc.

Muchiri, N., \& Kimathi, S. (2016). A review of applications and potential applications of UAV. Proc. Annual Conf. Sustainable Research and Innovation (pp. 280-283). Retrieved from http://sri.jkuat.ac.ke/ojs/index.php/proceedings/article/view/451

Pajares, G. (2015). Overview and current status of remote sensing applications based on unmanned aerial vehicles (UAVs). Photogram. Eng. Remote Sensing, 81(4), 281-329. https://doi.org/10.14358/PERS.81.4.281

Parkin, C. S., \& Wyatt, J. C. (1982). The determination of flightlane separations for the aerial application of herbicides. Crop Prot., 1(3), 309-321. https://doi.org/10.1016/02612194(82)90006-0

Qin, W.-C., Qiu, B.-J., Xue, X.-Y., Chen, C., Xu, Z.-F., \& Zhou, Q.-Q. (2016). Droplet deposition and control effect of insecticides sprayed with an unmanned aerial vehicle against plant hoppers. Crop Prot., 85, 79-88. https://doi.org/10.1016/j.cropro.2016.03.018

Quackenbush, T. R., Bliss, D. B., \& Wachspress, D. A. (1990). Free wake analysis of hover performance using a new influence coefficient method. NASA CR 4309. Washington, DC: National Aeronautics and Space Administration.

Quackenbush, T. R., Boschitsch, A. H., \& Wachspress, D. A. (1996). Fast analysis methods for surface-bounded flows with applications to rotor wake modeling. Proc. 52nd Annual Forum of the American Helicopter Society. Fairfax, VA: AHS International.

Quackenbush, T. R., Lam, C.-M. G., Chua, K., Bilanin, A. J., Bliss, D. B., \& Katz, A. (1991). Computational methods for the analysis of rotor wake/airframe interactions. Report No. 91-02. Ewing, NJ: Continuum Dynamics, Inc.

Quackenbush, T. R., Lam, C.-M. G., Wachspress, D. A., \& Bliss, D. B. (1994). Computational analysis of high-resolution unsteady airloads for rotor aeroacoustics. NASA CR 194894. Washington, DC: National Aeronautics and Space Administration.

Quackenbush, T. R., Wachspress, D. A., Boschitsch, A. H., \& Curbishley, T. B. (1999). A comprehensive hierarchical aeromechanics rotorcraft model (CHARM) for general rotor/surface interaction. Report No. 99-03. Ewing, NJ: Continuum Dynamics, Inc.

Quackenbush, T. R., Wachspress, D. A., Boschitsch, A. H., \& Keller, J. D. (2001). Real-time free wake methods for rotorcraft simulation and design. Report No. 01-11. Ewing, NJ: Continuum Dynamics, Inc.

Reed, W. H. (1953). An analytical study of the effect of airplane wake on the lateral dispersion of aerial sprays. NACA Report 3032. Langley, VA: National Advisory Committee for Aeronautics.

Tang, Q., Zhang, R. R., Chen, L. P., Xu, M., Yi, T. C., \& Zhang, B. (2017). Droplets movement and deposition of an eight-rotor agricultural UAV in downwash flow field. Intl. J. Agric. Biol. Eng., 10(3), 47-56. https://doi.org/10.3965/j.jjabe.20171003.3075

Teske, M. E. (2016). AGDISP DLL subroutine details, with application to Spray Advisor, SCIPUFF, and CALPUFF: AGDISP 8.29. Tech. Note No. 16-13. Ewing, NJ: Continuum Dynamics, Inc.

Teske, M. E., \& Barry, J. W. (1993). Parametric sensitivity in aerial application. Trans. ASAE, 36(1), 27-33. https://doi.org/10.13031/2013.28310
Teske, M. E., \& Thistle, H. W. (2003). Release height and far-field limits of Lagrangian aerial spray models. Trans. ASAE, 46(4), 977-983. https://doi.org/10.13031/2013.13953

Teske, M. E., \& Wachspress, D. A. (2017). The development of an AGDISP wake model for an unmanned aerial vehicle. Tech. Note No. 17-09. Ewing, NJ: Continuum Dynamics, Inc.

Teske, M. E., Bird, S. L., Esterly, D. M., Curbishley, T. B., Ray, S. L., \& Perry, S. G. (2002). AgDRIFT: A model for estimating near-field spray drift from aerial applications. Environ. Toxicol. Chem., 21(3), 659-671. https://doi.org/10.1002/etc.5620210327

Teske, M. E., Thistle, H. W., \& Ice, G. G. (2003). Technical advances in modeling aerially applied sprays. Trans. ASAE, 46(4), 985-996. https://doi.org/10.13031/2013.13955

Teske, M. E., Thistle, H. W., \& Londergan, R. J. (2011). Modification of droplet evaporation in the simulation of fine droplet motion using AGDISP. Trans. ASABE, 54(2), 417-421. https://doi.org/10.13031/2013.36444

von Kármán, T. D., \& Howarth, L. (1938). On the statistical theory of isotropic turbulence. Proc. Royal Soc. London A, 164(917), 192-215. https://doi.org/10.1098/rspa.1938.0013

Wachspress, D. A. (2015). Fully automated, agricultural application using unmanned aircraft: Phase I SBIR final report. Report No. 15-10. Ewing, NJ: Continuum Dynamics, Inc.

Wachspress, D. A., Keller, J. D., Quackenbush, T. R., Whitehouse, G. R., \& Yu, K. (2008). High-fidelity rotor aerodynamic module for real-time rotorcraft flight simulation. Proc. 64th Annual Forum of the American Helicopter Society. Fairfax, VA: AHS International.

Wachspress, D. A., Keller, J. D., Yu, K., \& Roark, S. (2012b). Next-generation flight simulation aerodynamic modeling for rotary wing aircraft. Report No. 12-08. Ewing, NJ: Continuum Dynamics, Inc.

Wachspress, D. A., Quackenbush, T. R., \& Boschitsch, A. H. (2003a). First principles free vortex wake analysis for helicopters and tiltrotors. Proc. 59th Annual Forum of the American Helicopter Society. Fairfax, VA: AHS International.

Wachspress, D. A., Quackenbush, T. R., \& Boschitsch, A. H. (2003b). Rotorcraft interactional aerodynamics with fast vortex/fast panel methods. J. American Helicopter Soc., 48(4), 223-235. https://doi.org/10.4050/JAHS.48.223

Wachspress, D. A., Whitehouse, G. R., Keller, J. D., Yu, K., Gilmore, P., Dorsett, M., \& McClure, K. (2009). A high-fidelity brownout model for flight simulations and trainers. Proc. 65th Annual Forum of the American Helicopter Society. Fairfax, VA: AHS International.

Wachspress, D. A., Yu, K., Saberi, H. A., Hasbun, M. J., Ho, J. C., \& Yeo, H. (2012a). Helicopter rotor airload predictions with a comprehensive rotorcraft analysis. Proc. 68th Annual Forum of the American Helicopter Society. Fairfax, VA: AHS International.

Wang, S. L., Song, J. L., He, X. K., Song, L., Wang, X. N., Wang, C. L., ... Ling, Y. (2017). Performances evaluation of four typical unmanned aerial vehicles used for pesticide application in China. Intl. J. Agric. Biol. Eng., 10(4), 22-31. https://doi.org/10.25165/j.ijabe.20171004.3219

Watts, A. C., Ambrosia, V. G., \& Hinkley, E. A. (2012). Unmanned aircraft systems in remote sensing and scientific research: Classification and considerations of use. Remote Sensing, 4(6), 1671-1692. https://doi.org/10.3390/rs4061671

Xue, X. Y., Tu, K., Qin, W. C., Lan, Y. B., \& Zhang, H. H. (2014). Drift and deposition of ultra-low altitude and low volume application in paddy field. Intl. J. Agric. Biol. Eng., 7(4), 23-28.

Xue, X., Lan, Y., Sun, Z., Chang, C., \& Hoffmann, W. C. (2016). Develop an unmanned aerial vehicle based automatic aerial spraying system. Comput. Electron. Agric., 128, 58-66. 